Journal of Mathematics and Statistics 6 (3): 271-275, 2010

ISSN 1549-3644

(C) 2010 Science Publications

\title{
On Certain Classes of Multivalent Analytic Functions
}

\author{
Rabha W. Ibrahim and Maslina Darus \\ Mathematics Program, School of Mathematical Sciences, \\ Faculty of Science and Technology, University Kebangsaan Malaysia Bangi 43600, \\ Selangor Darul Ehsan Malaysia, Malaysia
}

\begin{abstract}
Problem statement: By means of the Hadamard product (or convolution), new class of function of power order was formed. This class was motivated by many authors namely MacGregor, Umezawa, Darus and Ibrahim and many others. The class indeed extended in the form of integral operator due to the work of Bernardi, Libera and Livingston. Approach: A new class of multivalent analytic functions in the open unit disk $U$ was introduced. An application of this class was posed by using the fractional integral operator. The integral operator of multivalent functions was proposed and defined. The previous well known integral operator was mentioned. Results: Having the integral operator, a class was defined and coefficient bounds established by using standard method. These results reduced to well-known results studied by various authors. The operator was then applied for fractional calculus and obtained the coefficient bounds. Conclusion: Therefore, new operators could be obtained with some earlier results and standard methods. New classes were formed and new results of special cases were obtained.
\end{abstract}

Key words: Multivalent analytic function, Hadamard product, fractional integral operator, multiplier transformation, starlike function, convex function

\section{INTRODUCTION}

Let $\Sigma_{\mathrm{p}, \alpha}$ denote the class of functions of the form:

$\mathrm{f}(\mathrm{z})=\mathrm{z}^{\mathrm{p}+\alpha}+\sum_{\mathrm{n}=2}^{\infty} \mathrm{a}_{\mathrm{n}} \mathrm{z}^{\mathrm{n}+\mathrm{p}+\alpha},(0 \leq \alpha<1)$

which are analytic in the unit disk $\mathrm{U}:=\{\mathrm{z} \in \mathbb{C},|\mathrm{z}|<1\}$. For the Hadamard product or convolution of two power series $f$ defined in (1) and a function $g$ where:

$$
g(z)=z^{p+\alpha}+\sum_{n=2}^{\infty} b_{n} z^{n+p+\alpha},(z \in U)
$$

is:

$$
f(z) * g(z)=z^{p+\alpha}+\sum_{n=2}^{\infty} a_{n} b_{n} z^{n+p+\alpha},(z \in U)
$$

Also denote by $\mathrm{T}_{\mathrm{p}, \alpha}$ the subclass of $\Sigma_{\mathrm{p}, \alpha}$ consisting of functions of the form:

$f(z)=z^{p+\alpha}-\sum_{n=2}^{\infty}\left|a_{n}\right| z^{n+p+\alpha},(0 \leq \alpha<1)$ which are analytic in the unit disk U. For the Hadamard product or convolution of two power series f defined in (2) and a function $g$ where:

$$
g(z)=z^{p+\alpha}-\sum_{n=2}^{\infty}\left|b_{n}\right| z^{n+p+\alpha},(z \in U)
$$

is:

$$
f(z) * g(z)=z^{p+\alpha}-\sum_{n=2}^{\infty}\left|a_{n} \| b_{n}\right| z^{n+p+\alpha},(z \in U)
$$

Note that the authors defined and studied some classes of analytic functions take the form (1) and (2) (Darus and Ibrahim, 2008).

A function $\mathrm{f} \in \Sigma_{\mathrm{p} \alpha}$ is said to be in the class $\mathrm{P}_{\alpha}(\mathrm{p}, \mu)$, $(0<\mu<p+\alpha)$ if and only if it satisfies the inequality:

$$
\Re\left\{\frac{\mathrm{f}^{\prime}(\mathrm{z})}{\mathrm{z}^{\mathrm{p}+\alpha-1}}\right\}>\mu,(\mathrm{z} \in \mathrm{U})
$$

The classes $\mathrm{P}_{0}(1,0)$ and $\mathrm{P}_{0}(\mathrm{p}, 0)$ were investigated by MacGregor (1962) and Umezawa (1957), respectively.

Corresponding Author: Maslina Darus, Mathematics Program, School of Mathematical Sciences, Faculty of Science and Technology, University Kebangsaan Malaysia Bangi 43600, Selangor Darul Ehsan, Malaysia 


\section{MATERIALS AND METHODS}

In this study, we need to introduce a new integral operator such that certain classes can be defined by means of this integral operator. The previous operators will also be mentioned to highlight the importance of simple operator which then can be extended to a complicated ones and yet interesting to study.

For a function $f \in \Sigma_{p, \alpha}$ given by (1), we define the integral operator $J_{\alpha, p, c},(p, c \in \mathbb{N})$ and $(0 \leq \alpha<1)$ by:

$$
\begin{aligned}
J_{\alpha, p, c} f(z)= & \frac{c+p+\alpha}{z^{c}} \int_{0}^{z} t^{c-1} f(t) d t \\
& =z^{p+\alpha}+\sum_{n=2}^{\infty}\left[\frac{c+p+\alpha}{c+p+n+\alpha}\right] a_{n} z^{n+p+\alpha}
\end{aligned}
$$

Note that the operator $\mathrm{J}_{0,1, \mathrm{c}}$ was introduced by Bernardi (1969). In particular, the operator $\mathrm{J}_{0,1,1}$ was studied by Libera (1965) and Livingston (1966). Reddy and Padmanabhan (1982) defined the integral operator $\mathrm{J}_{0, \mathrm{p}, \mathrm{c}}$.

Clearly, (4) yields:

$$
\mathrm{f} \in \sum_{\mathrm{p}, \alpha} \Rightarrow \mathrm{J}_{\alpha, \mathrm{p}, \mathrm{c}} \in \sum_{\mathrm{p}, \alpha}
$$

Thus, by applying the operator $\mathrm{J}_{\alpha, \mathrm{p}, \mathrm{c}}$ successively, we can obtain the multiplier transformation:

$$
\begin{aligned}
J_{\alpha, p, c}^{k} f(z) & = \begin{cases}J_{\alpha, p, c}\left[J_{\alpha, p, c}^{k-1} f(z)\right], & (k \in \mathbb{N}) \\
f(z) & (k=0)\end{cases} \\
& =z^{p+\alpha}+\sum_{n=2}^{\infty}\left[\frac{c+p+\alpha}{c+p+n+\alpha}\right]^{k} a_{n} z^{n+p+\alpha}
\end{aligned}
$$

Cho (1993) defined and studied some subclasses involving the operator $\mathrm{J}_{0, \mathrm{p}, \mathrm{c}}^{\mathrm{k}}$.

A function $f \in \Sigma_{p, \alpha}$ is said to be $(p+\alpha)$-valent starlike of order $0 \leq \mu<p+\alpha$ if and only if:

$$
\Re\left\{\frac{\mathrm{zf}^{\prime}(\mathrm{z})}{\mathrm{f}(\mathrm{z})}\right\}>\mu,(\mathrm{z} \in \mathrm{U})
$$

We denote by $S_{p, \alpha}(\mu)$ the class of all such functions. A function $f \in \Sigma_{p, \alpha}$ is said to be $(p+\alpha)$-valent convex of order $0 \leq \mu<p+\alpha$ if and only if:

$$
\Re\left\{1+\frac{\mathrm{zf}^{\prime \prime}(\mathrm{z})}{\mathrm{f}^{\prime}(\mathrm{z})}\right\}>\mu,(\mathrm{z} \in \mathrm{U})
$$

Let $\mathrm{C}_{\mathrm{p}, \alpha}(\mu)$ denote the class of all those functions.
Note that $S_{1,0}(\mu)$ and $C_{1,0}(\mu)$ are, respectively, the usual classes of univalent starlike functions of order $\mu$ and univalent convex functions of order $\mu, 0 \leq \mu<1$. And $S_{1,0}(0)=S^{*}$ and $C_{1,0}(0)=C$ respectively which are the classes of univalent starlike (w.r.t. the origin) and univalent convex functions.

In the present study we define the subclass $S_{p, \alpha}(\mu, v)$ of $\Sigma_{p, \alpha}$ consisting of functions of the form (1) and satisfying the analytic criterion:

$$
\Re\left\{\frac{\mathrm{z}\left(\mathrm{J}_{\alpha, \mathrm{p}, \mathrm{c}}^{\mathrm{k}} \mathrm{f}(\mathrm{z})\right)^{\prime}}{\mathrm{J}_{\alpha, \mathrm{p}, \mathrm{c}}^{\mathrm{k}} \mathrm{f}(\mathrm{z})}-\mu\right\}>v\left|\frac{\mathrm{z}\left(\mathrm{J}_{\alpha, \mathrm{p}, \mathrm{c}}^{\mathrm{k}} \mathrm{f}(\mathrm{z})\right)^{\prime}}{\mathrm{J}_{\alpha, \mathrm{p}, \mathrm{c}}^{\mathrm{k}} \mathrm{f}(\mathrm{z})}-(\mathrm{p}+\alpha)\right|,(\mathrm{z} \in \mathrm{U})(5)
$$

for $0 \leq \mu<p+\alpha, v \geq 0$.

The main aim of this work is to study coefficient bounds and extreme points of the general subclass of $\Sigma_{\mathrm{p}, \alpha}$. Furthermore, we obtain special results.

\section{RESULTS}

Here we obtain a necessary and sufficient condition and extreme points for functions $f \in \Sigma_{\mathrm{p}, \alpha}$.

Theorem 1: Let $f \in \Sigma_{p, \alpha}$ A sufficient condition for a function of the form (1) to be in $S_{p, \alpha}(\mu, \nu)$ is that:

$$
\begin{gathered}
\sum_{n=2}^{\infty}\left[\frac{c+p+\alpha}{c+p+n+\alpha}\right]^{k}\left[\frac{n(1+v)+p+\alpha-\mu}{p+\alpha-\mu}\right]\left|a_{n}\right| \leq 1 \\
(z \in U)
\end{gathered}
$$

for $0 \leq \mu<p+\alpha, v \geq 0$.

Proof: Let $\mathrm{f}$ be of the form (1). It suffices to show that:

$$
\begin{aligned}
& v\left\{\frac{\mathrm{z}\left(\mathrm{J}_{\alpha, \mathrm{p}, \mathrm{c}}^{\mathrm{k}} \mathrm{f}(\mathrm{z})\right)^{\prime}}{\mathrm{J}_{\alpha, \mathrm{p}, \mathrm{c}}^{\mathrm{k}} \mathrm{f}(\mathrm{z})}-(\mathrm{p}+\alpha)\right\}- \\
& \Re\left|\frac{\mathrm{z}\left(\mathrm{J}_{\alpha, \mathrm{p}, \mathrm{c}}^{\mathrm{k}} \mathrm{f}(\mathrm{z})\right)^{\prime}}{\mathrm{J}_{\alpha, \mathrm{p}, \mathrm{c}}^{\mathrm{k}} \mathrm{f}(\mathrm{z})}-(\mathrm{p}+\alpha)\right| \leq \mathrm{p}+\alpha-\mu,(\mathrm{z} \in \mathrm{U})
\end{aligned}
$$

Yields:

$$
\begin{aligned}
& v\left|\frac{z\left(J_{\alpha, p, c}^{k} f(z)\right)^{\prime}}{J_{\alpha, p, c}^{k} f(z)}-(p+\alpha)\right|-\Re\left\{\frac{z\left(J_{\alpha, p, c}^{k} f(z)\right)^{\prime}}{J_{\alpha, p, c}^{k} f(z)}-(p+\alpha)\right\} \\
& \leq v\left|\frac{z\left(J_{\alpha, p, c}^{k} f(z)\right)^{\prime}}{J_{\alpha, p, c}^{k} f(z)}-(p+\alpha)\right|+\left|\frac{z\left(J_{\alpha, p, c}^{k} f(z)\right)^{\prime}}{J_{\alpha, p, c}^{k} f(z)}-(p+\alpha)\right| \\
& =(1+v)\left|\frac{z\left(J_{\alpha, p, c}^{k} f(z)\right)^{\prime}-(p+\alpha) J_{\alpha, p, c}^{k} f(z)}{J_{\alpha, p, c}^{k} f(z)}\right|
\end{aligned}
$$




$$
\begin{aligned}
& \leq \frac{(1+v) \sum_{n=2}^{\infty} n\left[\frac{c+p+\alpha}{c+p+n+\alpha}\right]^{k}\left|a_{n} \| z\right|^{n+p+\alpha}}{1-\sum_{n=2}^{\infty}\left[\frac{c+p+\alpha}{c+p+n+\alpha}\right]^{k}\left|a_{n} \| z\right|^{n+p+\alpha}} \\
& \leq \frac{(1+v) \sum_{n=2}^{\infty} n\left[\frac{c+p+\alpha}{c+p+n+\alpha}\right]^{k}\left|\alpha_{n}\right|}{1-\sum_{n=2}^{\infty}\left[\frac{c+p+\alpha}{c+p+n+\alpha}\right]^{k}\left|\alpha_{n}\right|}
\end{aligned}
$$

This last expression is bounded by $p+\alpha-\mu$ if:

$$
\begin{aligned}
& \sum_{n=2}^{\infty}\left[\frac{c+p+\alpha}{c+p+n+\alpha}\right]^{k} {[n(1+v)+p+\alpha-\mu] } \\
&\left|a_{n}\right| \leq p+\alpha-\mu,(z \in U)
\end{aligned}
$$

and the proof is complete.

Corollary 1: Let the assumptions of Theorem 1 hold. Then:

$$
\Re\left\{\frac{\mathrm{z}\left(\mathrm{J}_{\alpha, \mathrm{p}, \mathrm{c}}^{\mathrm{k}} \mathrm{f}(\mathrm{z})\right)^{\prime}}{\mathrm{J}_{\alpha, \mathrm{p}, \mathrm{c}}^{\mathrm{k}} \mathrm{f}(\mathrm{z})}\right\}>\mu
$$

Proof: By letting $v=0$.

Next we introduce some well known results which were studied by different authors.

Corollary 2: Let the assumptions of Theorem 1 hold. Then:

$$
\Re\left\{\frac{\mathrm{z}(\mathrm{f}(\mathrm{z}))^{\prime}}{\mathrm{f}(\mathrm{z})}\right\}>\mu
$$

Proof: By setting $\mathrm{k}=0, \mathrm{v}=0$.

Corollary 3: Let the assumptions of Theorem 1 hold. Then:

$$
\Re\left\{\frac{\mathrm{z}\left(\mathrm{J}_{\mathrm{p}, \mathrm{c}}^{\mathrm{k}} \mathrm{f}(\mathrm{z})\right)^{\prime}}{\mathrm{J}_{\mathrm{p}, \mathrm{c}}^{\mathrm{k}} \mathrm{f}(\mathrm{z})}\right\}>\mu
$$

Proof: By assuming $\alpha=0, v=0$

Corollary 4: Let the assumptions of Theorem 1 hold. Then:

$$
\Re\left\{\frac{\mathrm{z}(\mathrm{f}(\mathrm{z}))^{\prime}}{\mathrm{f}(\mathrm{z})}\right\}>0
$$

Proof: By setting $\mathrm{k}=0, \mu=v=0$.

Now we prove that the above condition is also necessary for $f \in T_{p, \alpha}$.

Theorem 2: A necessary and sufficient condition for $\mathrm{f}$ of the form (2) namely:

$$
f(z)-z^{p+\alpha}-\sum_{n=2}^{\infty} b_{n} z^{n+p+\alpha}, b_{n} \geq 0,(z \in U)
$$

to be in $\operatorname{TS}_{\mathrm{p}, \alpha}(\mu, v):=\mathrm{T}_{\mathrm{p}, \alpha} \cap \mathrm{S}_{\mathrm{p}, \alpha}(\mu, v), 0 \leq \mu<+\alpha, v \geq 0$ is that:

$$
\begin{array}{r}
\sum_{n=2}^{\infty}\left[\frac{c+p+\alpha}{c+p+n+\alpha}\right]^{k}[n(1+v)+p+\alpha-\mu] \\
b_{n} \leq p+\alpha-\mu,(z \in U)
\end{array}
$$

Proof: In view of Theorem 1 we need only to prove the necessity. If $f \in \mathrm{TS}_{\mathrm{p}, \alpha}(\mu, v)$ and $\mathrm{z}$ is real then:

$$
\begin{aligned}
& \frac{p+\alpha-\sum_{n=2}^{\infty}(n+p+\alpha)\left[\frac{c+p+\alpha}{c+p+n+\alpha}\right]^{k} b_{n} z^{n+p+\alpha}}{1-\sum_{n=2}^{\infty}\left[\frac{c+p+\alpha}{c+p+n+\alpha}\right]^{k} b_{n} z^{n+p+\alpha}}- \\
& \mu \geq \frac{v \sum_{n=2}^{\infty} n\left[\frac{c+p+\alpha}{c+p+n+\alpha}\right]^{k} b_{n} z^{n+p+\alpha}}{1-\sum_{n=2}^{\infty}\left[\frac{c+p+\alpha}{c+p+n+\alpha}\right]^{k} b_{n} z^{n+p+\alpha}} \Rightarrow \\
& p+\alpha-\mu-\sum_{n=2}^{\infty}\left[\frac{c+p+\alpha}{c+p+n+\alpha}\right]^{k}(n+p+\alpha-\mu) b_{n} \\
& >\sum_{n=2}^{\infty} v n>\left[\frac{c+p+\alpha}{c+p+n+\alpha}\right]^{k} b_{n}
\end{aligned}
$$

where, $z \rightarrow 1$ along the real axis, we obtain the desired inequality:

$$
\sum_{n=2}^{\infty}\left[\frac{c+p+\alpha}{c+p+n+\alpha}\right]^{k}[n(1+v)+p+\alpha-\mu] b_{n} \leq p+\alpha-\mu,(z \in U)
$$

Hence the proof.

Theorem 3: The extreme points of $\mathrm{TS}_{\mathrm{p}, \alpha}(\mu, v)$ are the functions given by:

$$
\begin{aligned}
& \mathrm{f}_{\mathrm{n}}(\mathrm{z})=\mathrm{z}^{\mathrm{p}+\alpha}- \\
& \frac{\mathrm{p}+\alpha-\mu}{\left[\frac{\mathrm{c}+\mathrm{p}+\alpha}{\mathrm{c}+\mathrm{p}+\mathrm{n}+\alpha}\right]^{\mathrm{k}}[\mathrm{n}(1+v)+\mathrm{p}+\alpha-\mu]} \mathrm{z}^{\mathrm{n}+\mathrm{p}+\alpha},(\mathrm{z} \in \mathrm{U})
\end{aligned}
$$


where, $n=2,3, \ldots, 0 \leq \mu<p+\alpha, v \geq 0$.

\section{DISCUSSION}

The class $\Sigma_{\mathrm{p}, \alpha}$ can further be applied in fractional calculus. For that reason, we need to the following definition.

Definition 1: The fractional integral of order $\alpha$ is defined, for a function $\mathrm{f}$ Srivastava and Owa (1989):

$$
\mathrm{I}_{\mathrm{z}}^{\alpha} \mathrm{f}(\mathrm{z}):=\frac{\mathrm{I}}{\Gamma(\alpha)} \int_{0}^{\mathrm{z}} \mathrm{f}(\zeta)^{\alpha-1} \mathrm{~d} \zeta ; \quad \alpha>0
$$

where, the function $\mathrm{f}$ is analytic in simply-connected region of the complex z-plane $(\mathbb{C})$ containing the origin and the multiplicity of $(\mathrm{z}-\zeta)^{\alpha-1}$ is removed by requiring $\log (\mathrm{z}-\zeta)$ to be real when $(\mathrm{z}-\zeta)>0$.

Note that (Srivastava and Owa, 1989; Miller and Ross, 1993):

$$
\mathrm{I}_{\mathrm{z}}^{\alpha} \mathrm{z}^{\mu}=\frac{\Gamma(\mu+1) \mathrm{I}}{\Gamma(\mu+1+\alpha)} \mathrm{z}^{\mu+\alpha},(\mu>-1)
$$

And some of its current properties can be found in (Ibrahim and Darus, 2008). For this purpose, let us denoted by $\Sigma_{\mathrm{p}}$ the class of functions $\mathrm{F}$ of the form:

$\mathrm{F}(\mathrm{z})=\phi_{1} \mathrm{z}^{\mathrm{p}}+\sum_{\mathrm{n}=2}^{\infty} \phi_{\mathrm{n}} \mathrm{z}^{\mathrm{n}+\mathrm{p}},\left(\phi_{1}=\frac{\Gamma(\alpha+2)}{\Gamma(2)}\right)$

which are analytic in the unit disk $U$. Let denoted by $T_{p}$ the class of functions $F$ of the form:

$\mathrm{F}(\mathrm{z})=\varphi_{1} \mathrm{z}^{\mathrm{p}}+\sum_{\mathrm{n}=2}^{\infty} \varphi_{\mathrm{n}} \mathrm{z}^{\mathrm{n}+\mathrm{p}},\left(\varphi_{1}=\frac{\Gamma(\alpha+2)}{\Gamma(2)}\right)$

$\varphi_{\mathrm{n}} \geq 0$ which are analytic in the unit disk $\mathrm{U}$.

Then in view of Definition 1, the fractional integral operator becomes:

$\mathrm{I}_{z}^{\alpha} \mathrm{F}(\mathrm{z})=\mathrm{z}^{\mathrm{P}+\alpha}+\sum_{\mathrm{n}=2}^{\infty} \mathrm{a}_{\mathrm{n}} \mathrm{z}^{\mathrm{n}+\mathrm{P}+\mathrm{a}},(\mathrm{z} \in \mathrm{U})$

where, $F \in \Sigma_{\mathrm{p}}, \mathrm{a}_{\mathrm{n}}:=\frac{\Gamma(\mathrm{n}+1) 1}{\Gamma(\mathrm{n}+\alpha+1)} \phi_{\mathrm{n}}$ That is for $\mathrm{F} \in \Sigma_{\mathrm{p}}$ implies $\mathrm{I}_{\mathrm{z}}^{\alpha} \mathrm{F}(\mathrm{z}) \in \Sigma_{\mathrm{p}, \alpha},(\mathrm{z} \in \mathrm{U})$.

Similarly for functions $F \in T_{p}$ we have:

$I_{z}^{\alpha} F(z)=z^{p+\alpha}-\sum_{n=2}^{\infty} b_{n} z^{n+p+\alpha},(z \in U)$ where, $\mathrm{F}(\mathrm{z}) \in \Sigma_{\mathrm{p}}, \mathrm{b}_{\mathrm{n}}:=\frac{\Gamma(\mathrm{n}+1) \mathrm{I}}{\Gamma(\mathrm{n}+\alpha+1)} \varphi_{\mathrm{n}} \geq 0$.

Thus in the similar manner of the proof of theorems 1 and 2 respectively, we can show the following results.

Theorem 4: Let $F \in \Sigma_{p}$. A sufficient condition for a function of the form (11) to be in $S_{p, \alpha}(\mu, v)$ is (6).

Theorem 5: Let $F \in T_{p}$, A necessary and sufficient condition for a function of the form (12) to be in $\operatorname{TS}_{\mathrm{p}, \alpha}(\mu, \nu)$ is (8).

Remark 1: For the above application, an inequality (5) becomes:

$\Re\left\{\frac{\mathrm{z}\left(\mathrm{J}_{\alpha, \mathrm{p}, \mathrm{I}}^{\mathrm{k}} \mathrm{I}_{\mathrm{z}}^{\alpha} \mathrm{F}(\mathrm{z})\right)^{\prime}}{\mathrm{J}_{\alpha, \mathrm{p}, \mathrm{c}}^{\mathrm{k}} \mathrm{I}_{\mathrm{z}}^{\alpha} \mathrm{F}(\mathrm{z})}-\mu\right\}>v\left|\frac{\mathrm{z}\left(\mathrm{J}_{\alpha, \mathrm{p}, \mathrm{c}}^{\mathrm{k}} \mathrm{I}_{z}^{\alpha} \mathrm{F}(\mathrm{z})\right)^{\prime}}{\mathrm{J}_{\alpha, \mathrm{p}, \mathrm{c}}^{\mathrm{k}} \mathrm{I}_{\mathrm{z}}^{\alpha} \mathrm{F}(\mathrm{z})}-(\mathrm{p}+\alpha)\right|,(\mathrm{z} \in \mathrm{U})$

for $0 \leq \mu<p+\alpha, v \geq 0$.

\section{CONCLUSION}

The work here concern mainly on multivalent analytic functions. This is not the only type of functions we are interested in. Recently, the meromorphic of fractional power has been studied as well. Many other results can be obtained through this fractional power by introducing different types of classes. Other problems to be considered include the coefficient estimates and the subordination problems.

\section{ACKNOWLEDGEMENT}

The work here is partially supported by MOHE: UKM-ST-06-FRGS0107-2009.

\section{REFERENCES}

Cho, N.E., 1993. Certain classes of p-valent analytic functions. Int. J. Math. Math. Sci., 16: 319-328. DOI: $10.1155 / \mathrm{S} 0161171293000389$

Bernardi, S.D., 1969. Convex and Starlike univalent functions. Trans. Am. Math. Soc., 135: 429-446. DOI: $10.2307 / 1995025$

Darus, M. and R. W. Ibrahim, 2008. Coefficient inequalities for a new class of univalent functions. Lobachevskii J. Math., 29: 221-229. DOI: 10.1134/S1995080208040045

Ibrahim, R.W. and M. Darus, 2008. Subordination and superordination for univalent solutions for fractional differential equations. J. Math. Anal. Appli., $\quad 345$ : 871-879. DOI: 10.1016/j.jmaa.2008.05.017 
Libera, R.J., 1965. Some classes of regular univalent functions. Proc. Am. Math. Soc., 16: 755-758. DOI: $10.2307 / 2033917$

Livingston, A.E., 1966. On the radius of univalence of certain analytic functions. Proc. Am. Math. Soc., 17: 352-357. DOI: $10.2307 / 2035165$

MacGregor, T.H., 1962. Functions whose derivatives has a positive real part. Trans. Am. Math. Soc., 104: 532-537. DOI: $10.2307 / 1993803$

Miller, K.S. and B. Ross, 1993. An Introduction to the Fractional Calculus and Fractional Differential Equations. 1st Edn., John-Wiley and Sons Inc., New York, ISBN: 10: 0471588849, pp: 384.
Reddy, G.L. and K.S. Padmanabhan, 1982. On analytic functions with reference to the Bernardi integral operator. Bull. Aust. Math. Soc., 25: 387-396. DOI: $10.1017 /$ S0004972700005438

Srivastava, H.M. and S. Owa, 1989. Univalent Functions, Fractional Calculus and Their Applications. 1st Edn., John Wiley and Sons Inc., New York, ISBN: 10: 0470216301, pp: 404.

Umezawa, T., 1957. Multivalently close-to-convex functions. Proc. Am. Math. Soc., 8: 869-874. DOI: $10.2307 / 2033681$ 